\title{
Efficacy and safety of cryoballoon ablation versus radiofrequency catheter ablation in atrial fibrillation: an updated meta-analysis
}

\author{
Honglan $\mathrm{Ma}^{1}$, Dongdong Sun ${ }^{2}$, Hui Luan ${ }^{1}$, Wei Feng ${ }^{3}$, Yaqiong Zhou ${ }^{1}$, Jine $\mathrm{Wu}^{1}$, Caiyun $\mathrm{He}^{4}$, Chaofeng Sun ${ }^{1}$ \\ ${ }^{1}$ Department of Cardiovascular Medicine, The First Affiliated Hospital of Xi'an Jiaotong University, Xi'an Shaanxi, China \\ ${ }^{2}$ Department of Cardiovascular Medicine, Capital Medical University Affiliated Beijing An Zhen Hospital, Beijing, China \\ ${ }^{3}$ Department of Respiratory and Critical Care Medicine, The First Affiliated Hospital of Xi'an Jiaotong University, Xi'an Shaanxi, China \\ ${ }^{4}$ Department of Endocrinology, The First Affiliated Hospital of Xi'an Jiaotong University, Xi'an Shaanxi, China
}

Adv Interv Cardiol 2017; 13, 3 (49): 240-249

DOI: https://doi.org/10.5114/aic.2017.70196

\begin{abstract}
A bstract
Introduction: Cryoballoon ablation (CBA) and irrigated radiofrequency catheter ablation (RFCA) are the main treatments for drug-refractory symptomatic atrial fibrillation (AF).

Aim: To compare the efficacy and safety between CBA and RFCA for the treatment of AF.

Material and methods: We searched the Embase and Medline databases for clinical studies published up to December 2016. Studies that satisfied our predefined inclusion criteria were included.

Results: After searching through the literature in the two major databases, 20 studies with a total of 9,141 patients were included in our study. The CBA had a significantly shorter procedure time (weighted mean difference (WMD) $-30.38 \mathrm{~min} ; 95 \% \mathrm{Cl}:-46.43$ to $-14.33, p=0.0002$ ) and non-significantly shorter fluoroscopy time (WMD $-3.18 \mathrm{~min} ; 95 \% \mathrm{Cl}:-6.43$ to $0.07, p=0.06)$ compared with RFCA. There was no difference in freedom from AF between CBA and RFCA (CBA 78.55\% vs. RFCA 83.13\%, OR $=1.15,95 \% \mathrm{Cl}$ : $0.95-1.39, p=0.14$ ). The CBA was associated with a high risk of procedure-related complications (CBA 9.02\% vs. RFCA 6.56\%, $\mathrm{OR}=1.56,95 \% \mathrm{Cl}: 1.05-2.31, p=0.03$ ), especially phrenic nerve paralysis ( $\mathrm{PNP}, \mathrm{OR}=10.72,95 \% \mathrm{Cl}: 5.59-20.55, p<0.00001$ ). The risk of pericardial effusions/cardiac tamponade was low in the CBA group (CBA 1.05\% vs. RFCA 1.86\%, OR $=0.62,95 \%$ CI: $0.41-0.93$, $p=0.02)$.

Conclusions: For AF, CBA was as effective as RFCA. However, CBA had a shorter procedure time and a non-significantly shorter fluoroscopy time, a significantly high risk of PNP and a low incidence of pericardial effusions/cardiac tamponade compared with RFCA.
\end{abstract}

Key words: cryoballoon ablation, radiofrequency catheter ablation, atrial fibrillation, meta-analysis.

\section{Introduction}

Atrial fibrillation (AF) is certainly the most frequent arrhythmia and is associated with an increased risk of stroke, heart failure, and mortality [1]. Today, cryoballoon ablation (CBA) is an acceptable alternative to point-bypoint radiofrequency catheter ablation (RFCA) for patients who are suffering from drug-refractory symptomatic AF [2]. However, there is still no universal agreement on which is preferable for treatment of AF. Although large numbers of clinical studies have already demonstrated CBA with a high success rate and low complication rate in the treatment of paroxysmal or persistent AF compared with RFCA $[3,4]$, it is still less clear whether CBA is superior to RFCA. Therefore, we intended to update a meta-analysis to compare the efficacy and safety of CBA and RFCA for pulmonary vein isolation (PVI) in patients with $A F$, and perform analyses in a subgroup to increase the specificity of our findings. All outcomes were assessed in accordance with definitions of the original protocols at the longest follow-up available.

\section{Aim}

This meta-analysis aimed to compare the efficacy and safety between CBA and RFCA for the treatment of AF.

\section{Corresponding author:}

Prof. Chaofeng Sun MD, PhD, Department of Cardiovascular Medicine, The First Affiliated Hospital of Xi'an Jiaotong University, Yanta Road No. 277, 710061 Xi'an, China, phone: +86 13700281636, e-mail: csun1@163.com

Received: 18.01.2017, accepted: 19.04.2017. 


\section{Material and methods}

Our study was carried out according to the guidelines published by the Preferred Reporting Items for Systematic Reviews and Meta-analyses (PRISMA) statement (Supplementary Checklist S1) [5].

\section{Search strategies}

We performed an online search for published studies using the databases of Embase and Medline up to December 2016 and identified all the studies which described the difference between CBA and RFCA in English. Our search strategy started with relevant keywords and medical subject heading terms including the following keywords: atrial fibrillation, AF, cryoballoon, cryoablation, cryoenergy, radiofrequency isolation, radiofrequency ablation, pulmonary vein isolation, PVI. Similar keywords were connected with "or" and dissimilar keywords were connected with "and". The reference list of all qualified studies was reviewed for identification of further relevant studies.

\section{Selection criteria}

The included studies had to fulfill the following criteria: 1. availability of data regarding procedure time, fluoroscopy time and total procedure-related complications with CBA vs. RFCA; 2. availability of data regarding the freedom from AF with CBA vs. RFCA; 3. the duration of follow-up should be $>6$ months after ablation with a 3-month blanking period; 4 . the number of patients should be at least 20 in each group.

The major exclusion criteria were as follows: 1 . the patients had previous ablation procedures; 2 . administration of antiarrhythmic drugs (AADs) during the follow-up after the blanking period; 3 . case reports, conference abstracts, review articles, and duplicated data should be excluded; 4 . follow-up of studies $<6$ months.

\section{Data extraction and quality assessment}

Two authors (Honglan Ma, Dongdong Sun) collected the data independently according to predefined search criteria. The third author (Hui Luan) would resolve the divergences. If available we entered the data into a standard Excel template: author \& year, procedure time, fluoroscopy time, ablation time, the patients' number of pericardial effusion/cardiac tamponade events, the patients' number of phrenic nerve paralysis (PNP) events, freedom from AF after the follow-up and so on. Freedom from bias was assessed for each study in accordance with The Cochrane Collaboration method [6].

\section{Outcome variables}

The primary outcomes were freedom from AF after the ablation at the end of follow-up, the total procedure time, fluoroscopy time, ablation time during the ablation procedures and redo ablation rate during the follow-up. Secondary outcomes were the procedure-related complications, which contained pericardial effusion/cardiac tamponade, stroke, PNP, pulmonary vein stenosis, atrioesophageal fistula or death. Pericardial effusion/cardiac tamponade and PNP were reported as stand-alone complications in CBA and RFCA.

\section{Statistical analysis}

We analyzed outcomes with the help of the Review Manager Version 5.3 software package (RevMan; The Cochrane Collaboration, Copenhagen, Denmark). We compared quantitative outcomes by weighted mean difference (WMD) and its 95\% confidence interval $(95 \% \mathrm{Cl})$ between cryoballoon and radiofrequency ablation procedures. We compared the qualitative outcomes by weighted odds ratio (OR) and the corresponding $95 \% \mathrm{Cl}$, which were counted by the Mantel-Haenszel method. Heterogeneity was assessed by $\chi^{2}$ test and quantified using the inconsistency index $\left(R^{2}\right)$ statistic, with $R^{2}$ values of $25 \%$, $25-50 \%$, or $50 \%$ signified as low, moderate, or high heterogeneity [6]. $P$-values $<0.05$ showed statistical significance.

\section{Results}

The process of study selection is summarized in Figure 1. Twenty studies [7-26] with a total of 9141 patients (CBA, $n=3659$ vs. RFCA, $n=5482$ ) were included in this meta-analysis. The baseline characteristics of included studies were illustrated in Table I. Four [8, 10, 11, 21] studies adopted a $28 \mathrm{~mm}$ cryoballoon, and the rest of the studies adopted mixed cryoballoons of $23 \mathrm{~mm}$ and $28 \mathrm{~mm}$. All included studies adopted the irrigated radiofrequency. All studies had patients matched for age, gender, atrial fibrillation duration, cardiovascular artery disease, hypertension, diabetes and left atrium diameter

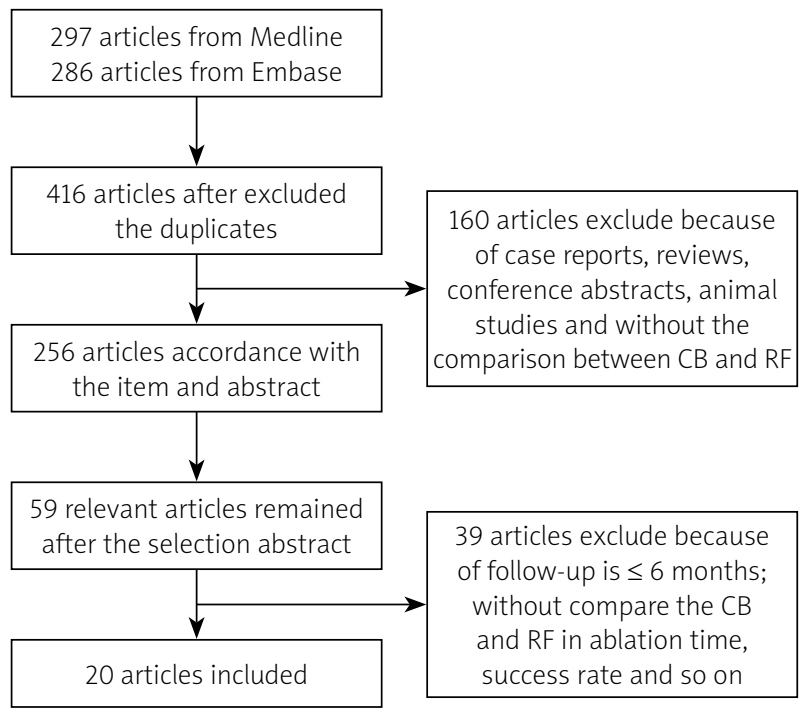

Figure 1. Flowchart of study selection procedure 


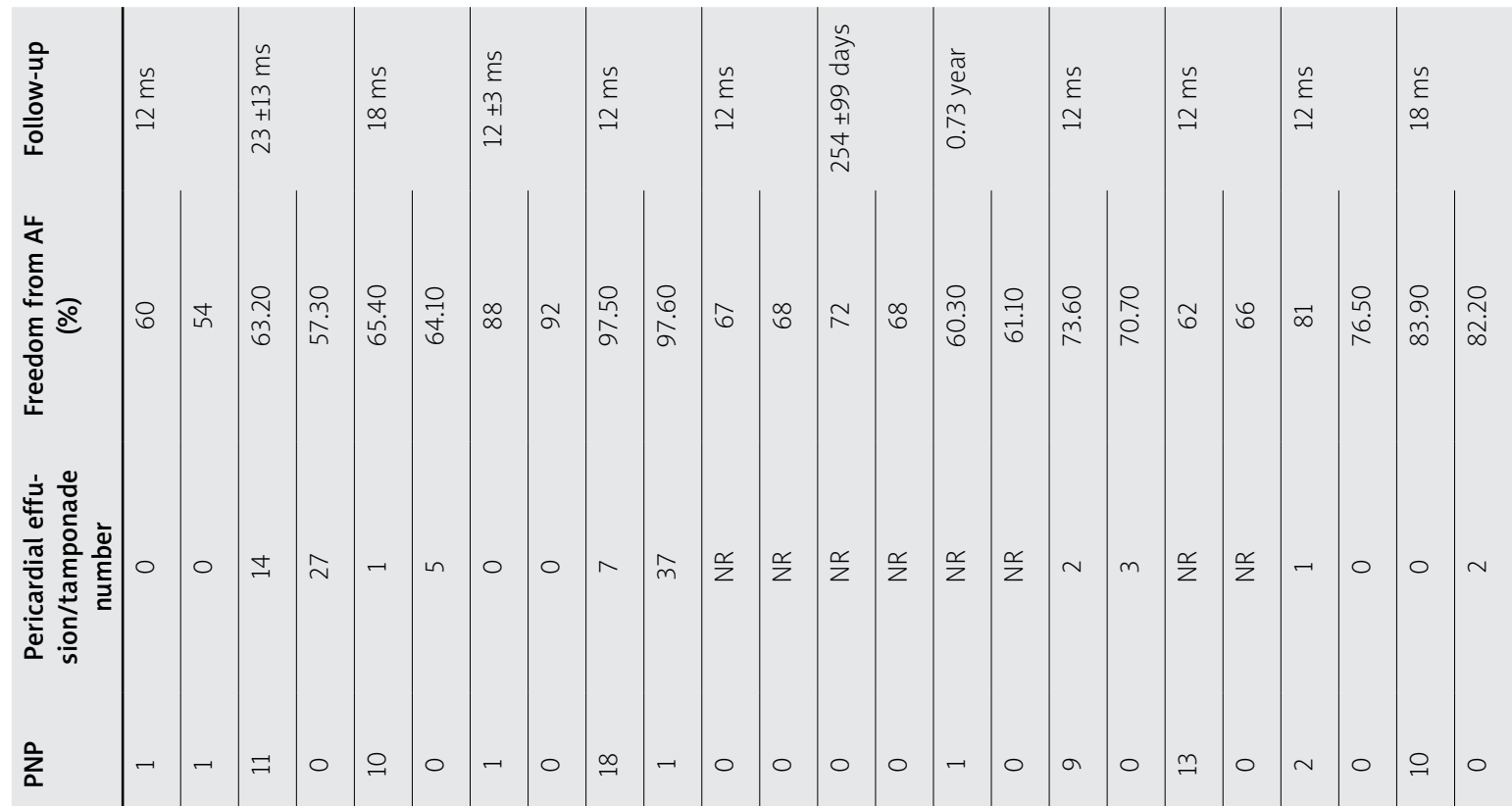

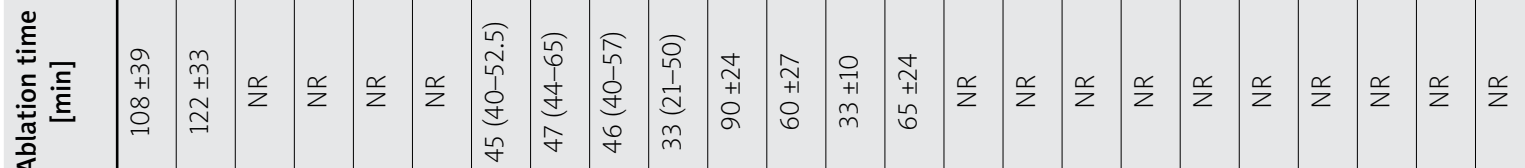

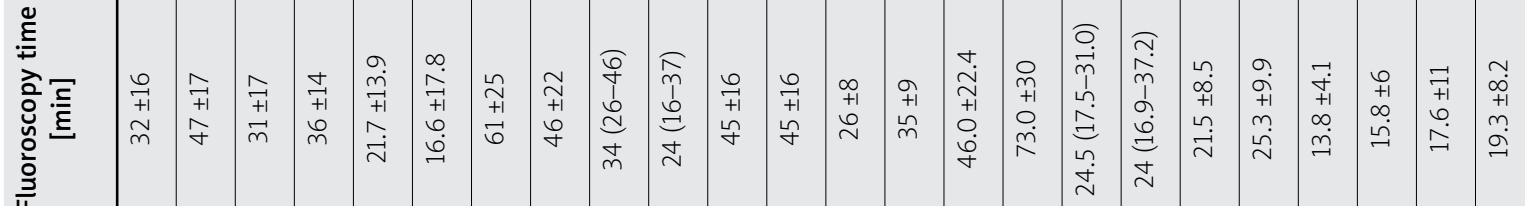

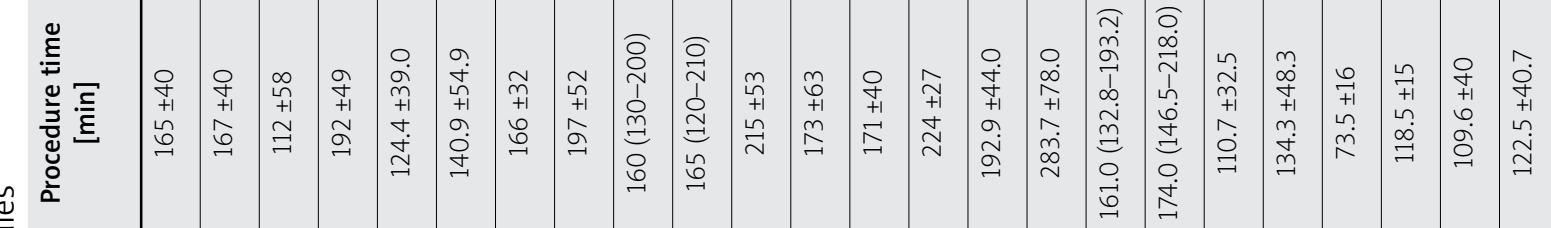
站

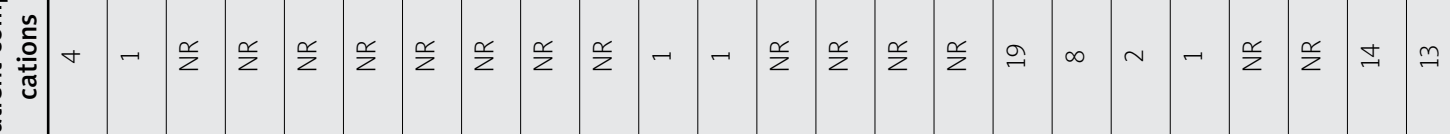

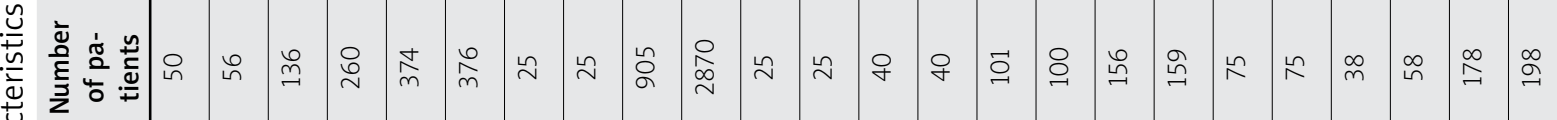
宅 完

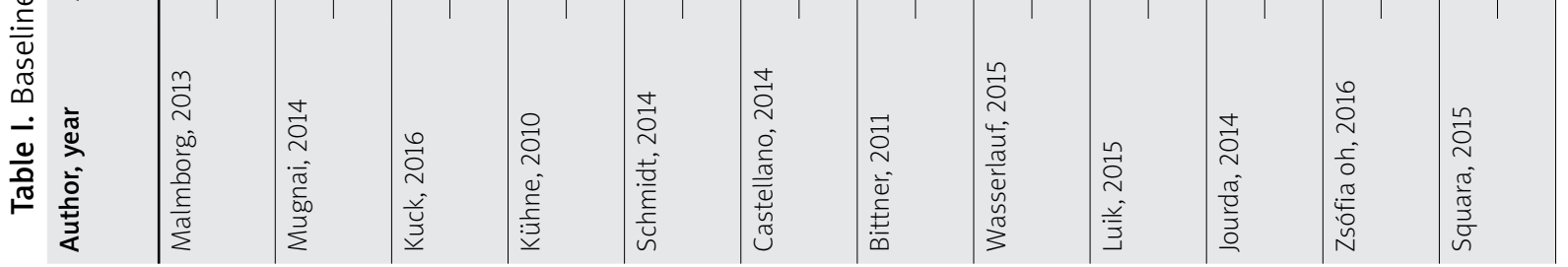




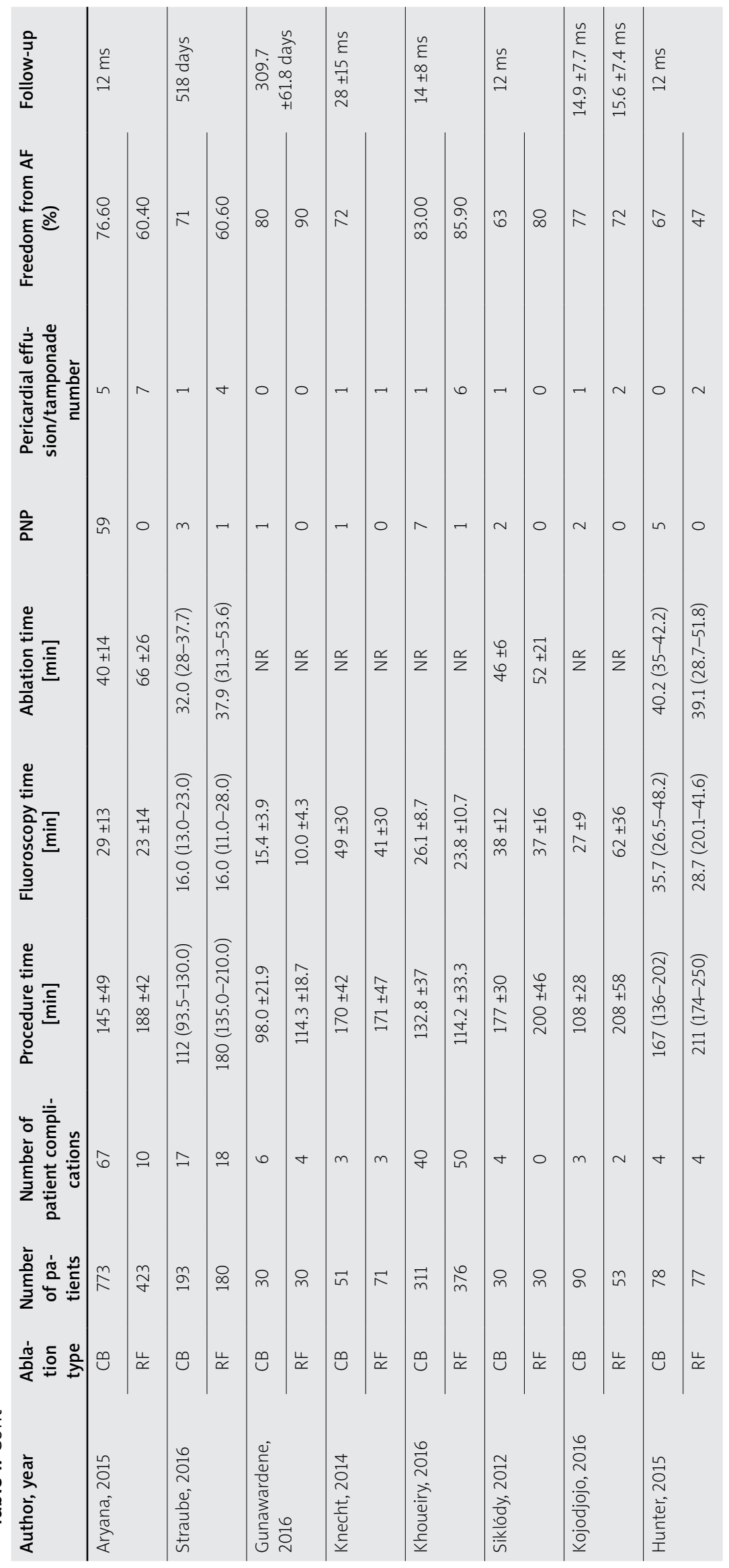


between CBA and RFCA according to the original design. Also, all studies described a blanking period of 3 months after ablation. No AADs were taken during the follow-up after the blacking period.

\section{Primary outcomes}

Our included data (20 studies, 9141 patients; CBA, $n=3659$ vs. RFCA, $n=5482$ ) showed similar efficacy in freedom from AF of $78.55 \%$ (2874 patients) for CBA compared to $83.13 \%$ (4557 patients) for RFCA (OR = 1.15, $95 \% \mathrm{Cl}: 0.95-1.39, p=0.14)$ with high heterogeneity for this risk estimate $\left(p=0.004, l^{2}=52 \%\right.$, Figure 2$)$. Four studies $[16,19,20,23]$ had been eliminated temporarily when comparing the procedure time and fluoroscopy time for not using the mean (standard deviation) to describe time. The procedure time (16 studies, 4523 patients) was significantly shorter among patients who underwent CBA than RFCA in our study (WMD -30.38 min; $95 \% \mathrm{Cl}:-46.43$ to $-14.33, p=0.0002$, Figure $3 \mathrm{~A}$ ). There was non-significantly shorter fluoroscopy time with CBA compared with RFCA (WMD $-3.18 \mathrm{~min} ; 95 \% \mathrm{Cl}$ : -6.43 to $0.07, p=0.06$, Figure $3 \mathrm{~B})$. There was high heterogeneity for this risk estimate in procedure time $(p<0.00001$, $\left.R^{2}=98 \%\right)$ and fluoroscopy time $\left(p<0.00001, l^{2}=95 \%\right)$. For redo ablation, CBA vs. RFCA was comparable during the follow-up after a blanking period of 3 months (WMD 0.83 ; $95 \% \mathrm{Cl}$ : 0.57-1.20, $p=0.31$ ).

\section{Secondary outcomes}

In our study, CB ablation was associated with relatively high risk of procedure-related complications (CBA $9.02 \%$ vs. RFCA $6.56 \%, \mathrm{OR}=1.56,95 \% \mathrm{Cl}: 1.05-2.31$, $p=0.03)$ with moderate heterogeneity for this risk estimate $\left(p=0.08, l^{2}=39 \%\right.$, Figure $\left.4 \mathrm{~A}\right)$. Pericardial effusion/ cardiac tamponade and PNP were reported as stand-alone complications. Fourteen studies with a total of 8504 patients recorded pericardial effusion/cardiac tamponade. There was a significant difference of 1.05\% (35 patients) for CBA compared to $1.86 \%$ (96 patients) for RFCA $(\mathrm{OR}=0.62,95 \% \mathrm{Cl}: 0.41-0.93, p=0.02)$ with no heterogeneity for this risk estimate $\left(p=0.65, l^{2}=0 \%\right.$, Figure $4 \mathrm{~B}$ ). Almost all PNP occurred in the CBA group during the procedure and follow-up period (CBA 156 patients vs. RFCA 4 patients). Therefore patients seemed more likely to obtain PNP in the CBA group than the RFCA group $(\mathrm{OR}=10.72,95 \% \mathrm{Cl}: 5.59-20.55, p<0.00001)$ after ablation with no heterogeneity for this risk estimate $(p=0.53$, $R^{2}=0 \%$, Figure $4 \mathrm{C}$ ). However, almost all PNP resolved during the procedure or within 1 year after ablation.

\section{Discussion}

This meta-analysis compared CBA with RFCA for the treatment of AF. The main findings were as follows: 1. CBA was associated with a significantly shorter procedure time and a non-significantly shorter fluoroscopy time than RFCA; both groups were comparable in the need for redo ablation; 2. CBA had a relatively high risk of procedure-related complications and a low risk of pericardial effusion/cardiac tamponade; 3 . There was no significant difference in freedom from $A F$ in a mean follow-up > 12 months between these two groups; 4. CBA had a higher risk of PNP than RFCA, although almost all PNP were resolved during the procedure and follow-up period.

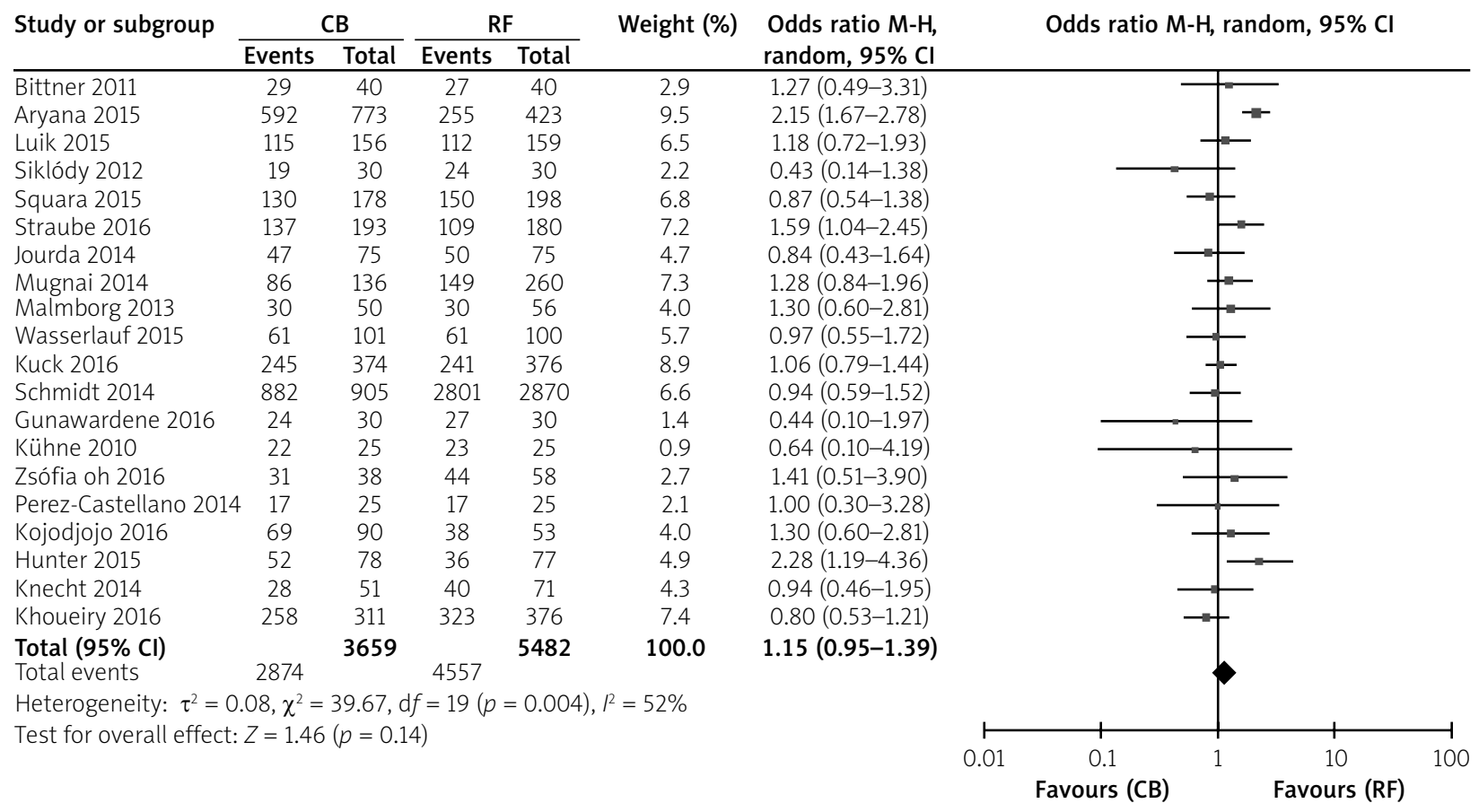

Figure 2. Forest plot of freedom from atrial fibrillation 
A. Comparison of procedure time

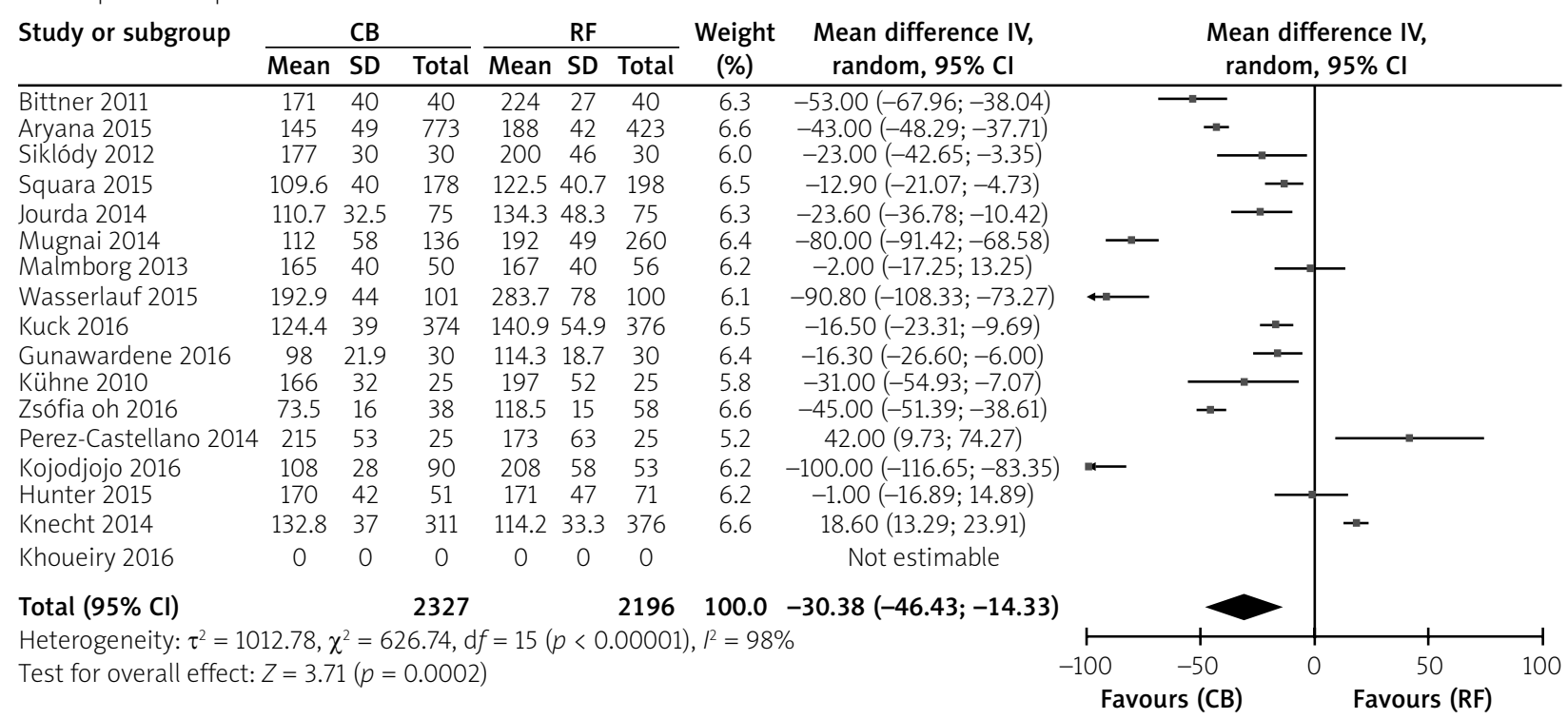

B. Comparison of fluoroscopy time

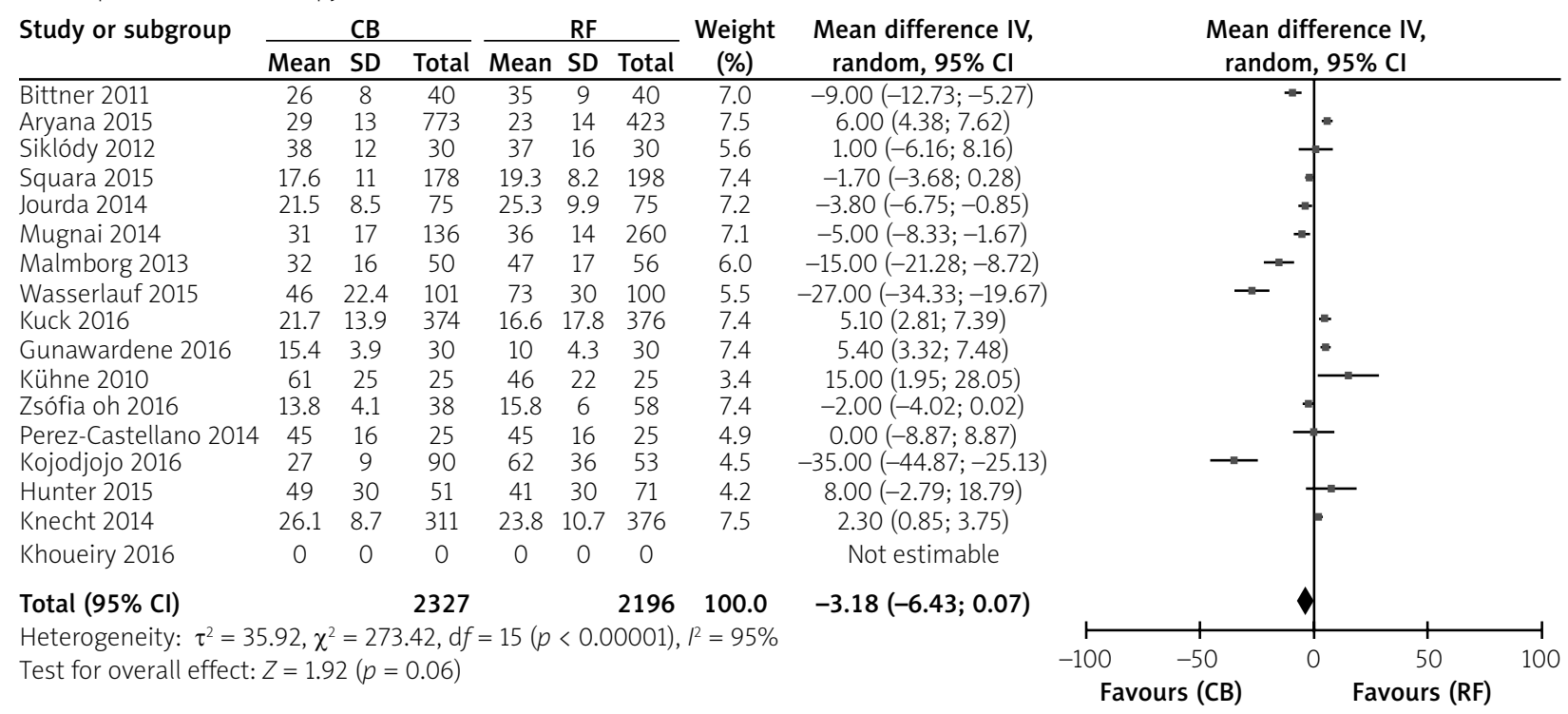

Figure 3. Forest plot of procedure and fluoroscopy time

Electrical isolation of the pulmonary veins (PVs) still remains the footstone of atrial fibrillation ablation procedures with the purpose of eliminating the trigger for AF episodes [27]. Radiofrequency and cryoballoon ablations are the two standard ablation systems used for PVI at present. Many experiments and studies have already compared the two standard ablations to determine which is more effective and safer. According to the previous studies $[28,29]$, the operators could use CBA or RFCA for AF patients because of the similar efficacy and safety, but evidence showed that CBA had a shorter learning curve than RFCA [30]. Hence we expect that CBA may be the main isolation in the future for $A F$ because of the short learning curve. Furthermore, previous studies $[31,32]$ have reported that the second-generation cryo- balloon ablation had a higher success rate than the first generation, and contact force ablation would reduce the major complications compared to non-contact force ablation [33]. However, just four [18, 21, 24, 26] of our studies stated that they used the second-generation cryoballoon ablation, and two studies $[14,19]$ stated that they used the first-generation counterpart.

As in previous studies, there was no significant difference in freedom from AF between CBA and RFCA during the follow-up in our study (CBA 78.55\% vs. RFCA 83.13\%, $p=0.14)$. Some published studies $[9,18,34]$ reported that freedom from AF after ablation ranged from $50 \%$ to $80 \%$. However, one study [16] showed a higher success rate of both CBA (97.5\%) and RFCA (97.6\%) groups compared to other studies. This may relate to it being a multi- 
A. Comparison of total procedure related complications Study or subgroup $\frac{\mathrm{CB}}{\text { Events Total }} \frac{\mathrm{RF}}{\text { Events Total }}$ Weight (\%) Odds ratio $\mathrm{M}-\mathrm{H}, \quad$ Odds ratio $\mathrm{M}-\mathrm{H}$, random, $95 \% \mathrm{Cl}$

\begin{tabular}{l}
\hline Aryana 2015 \\
Luik 2015 \\
Siklódy 2012 \\
Squara 2015 \\
Straube 2016 \\
Jourda 2014 \\
Malmborg 2013 \\
Gunawardene 2016 \\
Zsófia oh 2016 \\
Kojodjojo 2016 \\
Hunter 2015 \\
Knecht 2014 \\
Khoueiry 2016
\end{tabular}

$\begin{array}{cc}67 & 773 \\ 19 & 156 \\ 4 & 30 \\ 14 & 178 \\ 17 & 193 \\ 2 & 75 \\ 4 & 50 \\ 6 & 30 \\ 1 & 25 \\ 3 & 90 \\ 4 & 78 \\ 3 & 51 \\ 40 & 311\end{array}$

$\begin{array}{cc}10 & 423 \\ 8 & 159 \\ 0 & 30 \\ 13 & 198\end{array}$

$14.3 \quad 3.92(1.99-7.70)$

$\begin{array}{cc}11.4 & 2.62(1.11-6.17) \\ 1.7 & 10.36(0.53-201.45)\end{array}$

$1.7 \quad 10.36(0.53-201.45)$

$13.9 \quad 0.87(0.43-1.74)$

$2.4 \quad 2.03(0.18-22.85)$

$2.8 \quad 4.78(0.52-44.30)$

$\begin{array}{ll}2.8 & 4.78(0.52-44.30) \\ 6.1 & 1.63(0.41-6.47)\end{array}$

$1.8 \quad 1.00(0.06-16.93)$

$3.90 .88(0.14-5.44)$

$5.9 \quad 0.99(0.24-4.09)$

$4.7 \quad 1.42(0.27-7.32)$

Khoueiry 2016

$18.7 \quad 0.96(0.62-1.50)$

\section{Total $(95 \%$ C)}

2040

$50 \quad 376$

Total events

$184 \quad 115$

1753

100.0

$1.56(1.05-2.31)$

Heterogeneity: $\tau^{2}=0.17, \chi^{2}=19.53, \mathrm{~d} f=12$ ( $\left.p=0.08\right), \rho^{2}=39 \%$

Test for overall effect: $Z=2.19(p=0.03)$

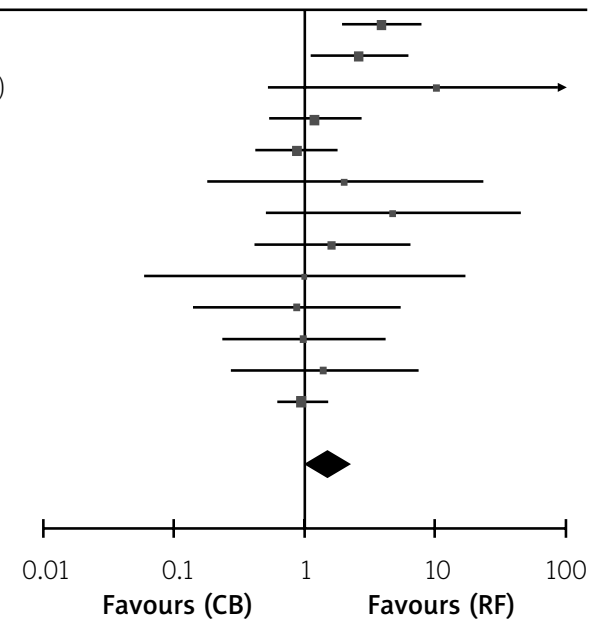

B. Comparison of pericardial effusion/cardiac tamponade

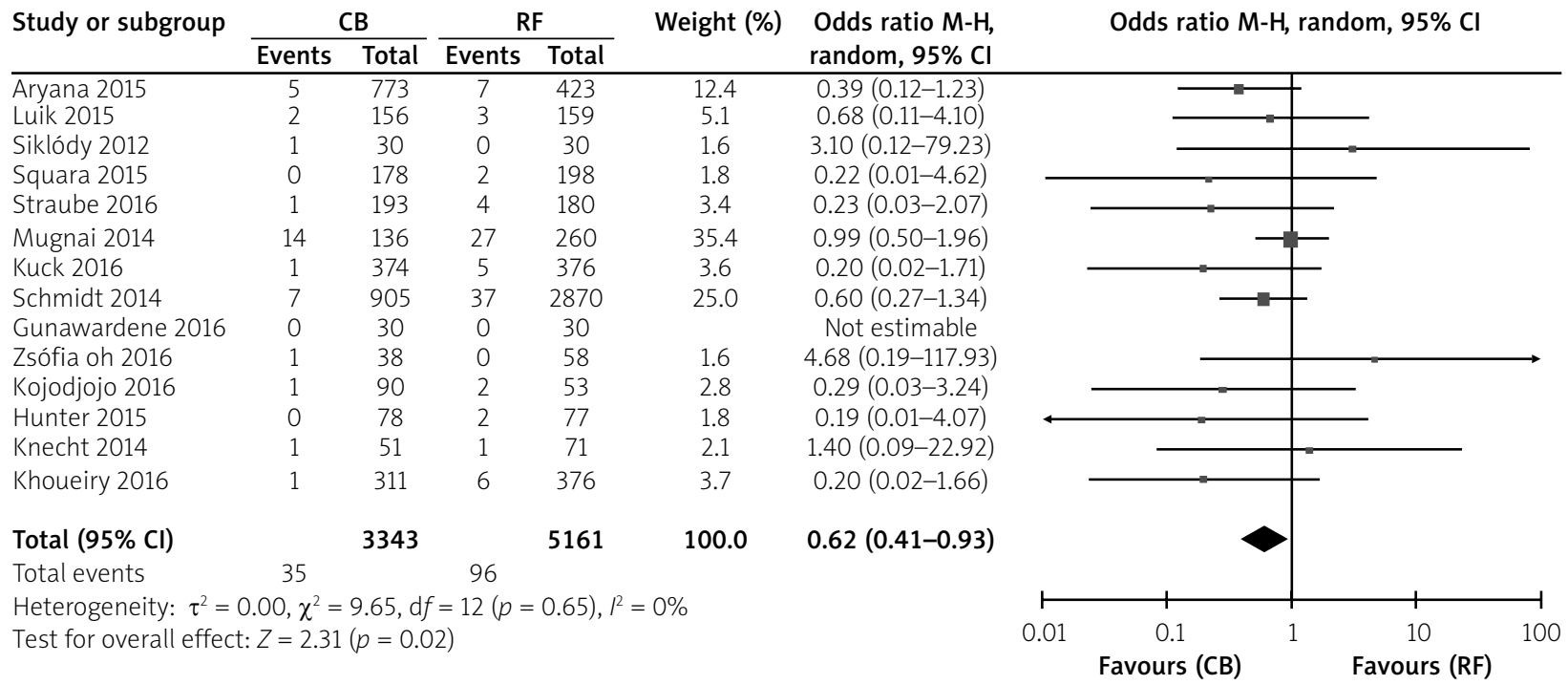

C. Comparison of PNP

Study or subgroup $\frac{\mathrm{CB}}{\text { Events Total }} \frac{\mathrm{RF}}{\text { Events Total }}$

Weight (\%) Odds ratio $\mathrm{M}-\mathrm{H}$, random, $95 \% \mathrm{Cl}$

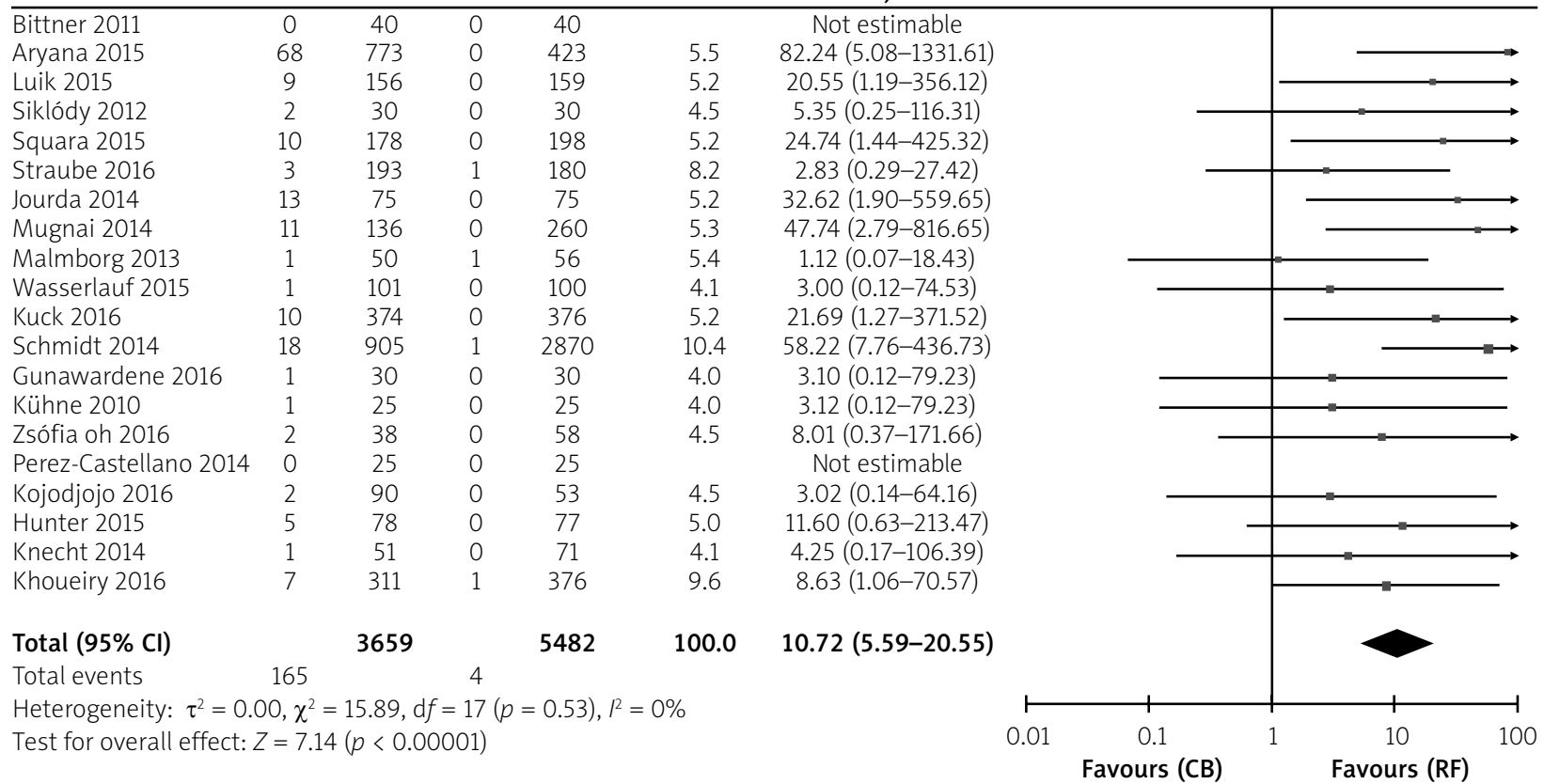

Figure 4. Forest plot of different procedure-related complications 
center prospective registry including a total of 55 German electrophysiological centers with vast experience. Also it was possibly due to methodological limitations including inadequate sample size, patient's section, and lack of adjustment for confounder bias.

In our meta-analysis, CBA had a shorter procedure time $(p=0.0002)$ and a non-significantly shorter fluoroscopy time $(p=0.06)$ compared with RFCA. The results were in line with most recent studies. As we know, the operators selected either CBA or RFCA on the basis of the patient's pulmonary vein anatom; in other words, patients with unfavorable anatomy on computed tomography may tend to have RFCA rather than CBA [35]. This may explain why most recent studies reported a shorter procedure time and fluoroscopy time in CBA. Data from the German Ablation Registry showed similar procedure times between CBA and RFCA, but fluoroscopy times were longer in CBA [16]. This difference was likely due to the need for pre-procedural computed tomography imaging, which can further increase the cumulative radiation dose received by the patients. It may also be explained by the different cumulative experience and technical skills at different centers. Additional focal ablation was used (either cryoballoon or RF-based) when PV isolation had not been finished alone in most studies. In our study there was no difference in the need for redo ablation during the follow-up between CBA and RFCA $(p=0.31)$. The increase of operator experience, technical skills and combined ablation might reduce the procedure time, fluoroscopy time and the need for redo ablation whether in CBA or RFCA. We speculate that with the accumulation of operational experience, cryoballoon ablation's advantages over radiofrequency ablation for the treatment of $A F$ will render it more and more viable in clinical practice.

The total procedure-related complication rate was higher in the CBA than the RFCA group (CBA 9.02\% vs. RFCA 6.56\%, $p=0.03$ ), especially the PNP ( $p<0.00001)$. As we all know, PNP occurred disproportionately more often in CBA compared with RFCA because of the mechanism of $C B$ ablation as well as the pulmonary vein and phrenic nerve anatomy. The high incidence of PNP in CB ablation accounts for a larger surface area of coolant distribution with $\mathrm{CB}$ catheters, which allows a more extended and simultaneous circumferential ablation with the potential reduction of bonus freeze cycle(s) and/or additional touch-up applications [36]. According to known anatomy, the phrenic nerves are relatively close to the pulmonary veins, especially the right phrenic nerve. Sanchez-Quintana et al. [37] reported that the right phrenic nerve was in close proximity to the superior caval vein (minimum: $0.3 \pm 0.5 \mathrm{~mm}$ ) and the right superior pulmonary vein (minimum: $2.1 \pm 0.4 \mathrm{~mm}$ ), which may enhance the risk of having PNP during ablation, but almost all PNP resolved during the procedure and follow-up period. Although extensive operator experience and high technical skills could decrease the incidence of PNP, additional ECG conduction is an easy, effective and helpful additional security measure to prevent PNP from happening in CBA [38].

In previous studies $[8,9,18,39]$, no significant differences were found between CBA and RFCA-related pericardial effusions/cardiac tamponade. In the FIRE and ICE trial [25], there were 5 (1.3\%) pericardial effusions with RFCA compared to $1(0.3 \%)$ in CBA $(p=0.22)$. Chierchia et al. [40] observed that pericardial effusion occurred in a similar proportion following CBA (11\%) and RFCA (16\%) for AF, whereas in our meta-analysis, RFCA showed a high risk of pericardial effusions/cardiac tamponade (CBA $1.05 \%$ vs. RFCA $1.86 \%, p=0.02$ ) compared to CBA. However, pericardial effusion/cardiac tamponade was mostly mild and asymptomatic, with benign clinical outcomes not requiring additional hospitalization days.

Despite the strengths of our study including a huge number of patients, and low risk of publication bias, there were still several limitations. Firstly, just several studies were designed in receiver operating characteristic curve, which might increase the selection bias. Secondly, according to the selection criteria, small trials (number of patients <20) and non-English published studies were excluded. Also, we did not attempt to include unpublished data such as those presented at scientific meetings in abstract form. Thirdly, data of procedure and fluoroscopy times were not all recorded as mean (standard deviation). Four studies (4618 patients) were excluded when comparing the procedure and fluoroscopy times. Just 13 studies (3793 patients) recorded the data of the procedure-related complications. Almost all included studies did not record the institutional level of experience (no. ablations/ year) with either ablation catheter.

\section{Conclusions}

The results of our meta-analysis showed that $C B$ ablation had a shorter procedure time and non-significantly shorter fluoroscopy time compared with RF ablation. There was no difference in freedom from AF after ablation at the end of follow-up between CBA and RFCA. But the total procedure-related complication rate was higher in CB ablation, especially the PNP. At the same time, our study indicated that RF ablation is associated with a high risk of pericardial effusions/cardiac tamponade.

\section{Acknowledgments}

This study was supported by the Clinical Research Award of the First Affiliated Hospital of Xi'an Jiaotong University, China (No. XJTU1AF-CRF-2015-007). Honglan $\mathrm{Ma}$ and Dongdong Sun contributed equally to this work.

\section{Conflict of interest}

The authors declare no conflict of interest. 


\section{References}

1. Benjamin EJ, Wolf PA, D'Agostino RB, et al. Impact of atrial fibrillation on the risk of death the Framingham Heart Study. Circulation 1998; 98: 946-52.

2. Packer DL, Kowal RC, Wheelan KR, et al. Cryoballoon ablation of pulmonary veins for paroxysmal atrial fibrillation: first results of the North American Arctic Front (STOP AF) pivotal trial. J Am Coll Cardiol 2013; 61: 1713-23.

3. Vogt J, Heintze J, Gutleben KJ, et al. Long-term outcomes after cryoballoon pulmonary vein isolation: results from a prospective study in 605 patients. J Am Coll Cardiol 2013; 61: 1707-12.

4. Defaye P, Kane A, Chaib A, et al. Efficacy and safety of pulmonary veins isolation by cryoablation for the treatment of paroxysmal and persistent atrial fibrillation. Europace 2011; 13: 789-95.

5. Vrabel M. Preferred reporting items for systematic reviews and meta-analyses. Oncol Nurs Forum 2015; 42: 552-4.

6. Higgins JP, Thompson SG, Deeks JJ, Altman DG. Measuring inconsistency in meta-analysis. BMJ 2003; 327: 557-60.

7. Malmborg H, Lonnerholm S, Blomstrom P, et al. Ablation of atrial fibrillation with cryoballoon or duty-cycled radiofrequency pulmonary vein ablation catheter: a randomized controlled study comparing the clinical outcome and safety; the AF-COR study. Europace 2013; 15: 1567-73.

8. Mugnai G, Chierchia GB, De Asmundis C, et al. Comparison of pulmonary vein isolation using cryoballoon versus conventional radiofrequency for paroxysmal atrial fibrillation. Am J Cardiol 2014; 113: 1509-13.

9. Kuck KH, Brugada J, Fürnkranz A, et al. Cryoballoon or radiofrequency ablation for paroxysmal atrial fibrillation. $N$ Engl I Med 2016; 374: 2235-45.

10. Kojodjojo P, O'Neill MD, Lim PB, et al. Pulmonary venous isolation by antral ablation with a large cryoballoon for treatment of paroxysmal and persistent atrial fibrillation: medium-term outcomes and non-randomised comparison with pulmonary venous isolation by radiofrequency ablation. Heart 2010; 96 : 1379-84.

11. Kuhne $M$, Suter $Y$, Altmann D, et al. Cryoballoon versus radiofrequency catheter ablation of paroxysmal atrial fibrillation: biomarkers of myocardial injury, recurrence rates, and pulmonary vein reconnection patterns. Heart Rhythm 2010; 7: 1770-6.

12. Bittner A, Monnig G, Zellerhoff S, et al. Randomized study comparing duty-cycled bipolar and unipolar radiofrequency with point-by-point ablation in pulmonary vein isolation. Heart Rhythm 2011; 8: 1383-90.

13. Herrera Siklody C, Arentz T, Minners J, et al. Cellular damage, platelet activation, and inflammatory response after pulmonary vein isolation: a randomized study comparing radiofrequency ablation with cryoablation. Heart Rhythm 2012; 9: 189-96.

14. Knecht S, Sticherling C, Von Felten S, et al. Long-term comparison of cryoballoon and radiofrequency ablation of paroxysmal atrial fibrillation: a propensity score matched analysis. Int J Cardiol 2014; 176: 645-50.

15. Perez-Castellano N, Fernandez-Cavazos R, Moreno J, et al. The COR trial: a randomized study with continuous rhythm monitoring to compare the efficacy of cryoenergy and radiofrequency for pulmonary vein isolation. Heart Rhythm 2014; 11: 8-14.

16. Schmidt M, Dorwarth U, Andresen D, et al. Cryoballoon versus RF ablation in paroxysmal atrial fibrillation: results from the German Ablation Registry. J Cardiovasc Electrophysiol 2014; 25: 1-7.
17. Wasserlauf J, Passman R, Giedrimas E, et al. Cryoballoon versus radiofrequency catheter ablation for atrial fibrillation. J Cardiovasc Electrophysiol 2014; 25: 568-9.

18. Aryana A, Singh SM, Kowalski M, et al. Acute and long-term outcomes of catheter ablation of atrial fibrillation using the second-generation cryoballoon versus open-irrigated radiofrequency: a multicenter experience. J Cardiovasc Electrophysiol 2015; 26: 832-9.

19. Hunter RJ, Baker V, Finlay MC, et al. Point-by-point radiofrequency ablation versus the cryoballoon or a novel combined approach: a randomized trial comparing 3 methods of pulmonary vein isolation for paroxysmal atrial fibrillation (The Cryo Versus RF Trial). J Cardiovasc Electrophysiol 2015; 26: 1307-14.

20. Luik A, Radzewitz A, Kieser M, et al. Cryoballoon versus open irrigated radiofrequency ablation in patients with paroxysmal atrial fibrillation: the prospective, randomized, controlled, noninferiority freeze AF study. Circulation 2015; 132: 1311-9.

21. Gunawardene MA, Hoffmann BA, Schaeffer B, et al. Influence of energy source on early atrial fibrillation recurrences: a comparison of cryoballoon vs. radiofrequency current energy ablation with the endpoint of unexcitability in pulmonary vein isolation. Europace 2016 Oct 14 pii: euw307 [Epub ahead of print].

22. Oh NZ, Zsuzsanna K, Zoltán S, et al. Catheter ablation for paroxysmal atrial fibrillation: new generation cryoballoon or contact force sensing radiofrequency ablation? Orvosi Hetilap 2016; 157: 849-54.

23. Straube F, Dorwarth U, Ammar-Busch S, et al. First-line catheter ablation of paroxysmal atrial fibrillation: outcome of radiofrequency vs. cryoballoon pulmonary vein isolation. Europace 2016; 18: 368-75.

24. Squara F, Zhao A, Marijon E, et al. Comparison between radiofrequency with contact force-sensing and second-generation cryoballoon for paroxysmal atrial fibrillation catheter ablation: a multicentre European evaluation. Europace 2015; 17: 718-24.

25. Kuck KH, Fürnkranz A, Chun KRJ, et al. Cryoballoon or radiofrequency ablation for symptomatic paroxysmal atrial fibrillation: reintervention, rehospitalization, and quality-of-life outcomes in the FIRE and ICE trial. Eur Heart J 2016; 37: 2858-65.

26. Jourda F, Providencia R, Marijon E, et al. Contact-force guided radiofrequency vs. second-generation balloon cryotherapy for pulmonary vein isolation in patients with paroxysmal atrial fibrillation - a prospective evaluation. Europace 2015; 17: 225-31.

27. Calkins H, Kuck KH, Cappato R, et al. 2012 HRS/EHRA/ECAS Expert Consensus Statement on Catheter and Surgical Ablation of Atrial Fibrillation: recommendations for patient selection, procedural techniques, patient management and follow-up, definitions, endpoints, and research trial design. Europace 2012; 14: 528-606.

28. Ang R, Domenichini G, Finlay MC, et al. The hot and the cold: radiofrequency versus cryoballoon ablation for atrial fibrillation. Curr Cardiol Rep 2015; 17: 631.

29. Cheng X, Hu Q, Zhou C, et al. The long-term efficacy of cryoballoon vs irrigated radiofrequency ablation for the treatment of atrial fibrillation: a meta-analysis. Int J Cardiol 2015; 181: 297-302.

30. Wójcik M, Berkowitsch A, Greis H, et al. Learning curve in cryoballoon ablation of atrial fibrillation. Circ J 2014; 78: 1612-8.

31. Conti S, Moltrasio M, Fassini G, et al. Comparison between firstand second-generation cryoballoon for paroxysmal atrial fibrillation ablation. Cardiol Res Pract 2016; 2016: 5106127. 
32. Pandya B, Sheikh A, Spagnola J, et al. Safety and efficacy of second-generation versus first-generation cryoballoons for treatment of atrial fibrillation: a meta-analysis of current evidence. J Interv Cardiac Electrophysiol 2016; 45: 49-56.

33. Akca F, Janse P, Theuns DA, et al. A prospective study on safety of catheter ablation procedures: contact force guided ablation could reduce the risk of cardiac perforation. Int I Cardiol 2015; 179: 441-8.

34. Aksu T, Guler TE, Golcuk SE, et al. Combined use of cryoballoon and radio frequency ablation for treatment of long-standing persistent atrial fibrillation: a single center experience. Am J Cardiol 2016; 117: S1.

35. Knecht S, Kuhne M, Altmann D, et al. Anatomical predictors for acute and mid-term success of cryoballoon ablation of atrial fibrillation using the $28 \mathrm{~mm}$ balloon. J Cardiovasc Electrophysiol 2013; 24: 132-8.

36. Metzner A, Wissner E, Ouyang F, et al. Second-generation cryoballoon ablation for paroxysmal atrial fibrillation: A step forward? Europace 2014: 16: 621-2.

37. Sanchez-Quintana D, Cabrera JA, Climent V, et al. How close are the phrenic nerves to cardiac structures? Implications for cardiac interventionalists. J Cardiovasc Electrophysiol 2005; 16: 309-13.

38. Meissner A, Maagh P, Christoph A, et al. ECG-guided surveillance technique in cryoballoon ablation for paroxysmal and persistent atrial fibrillation: a strategy to prevent from phrenic nerve palsy. Int J Med Sci 2016; 13: 403-11.

39. Luik A, Radzewitz A, Kieser M, et al. Cryoballoon versus open irrigated radiofrequency ablation in patients with paroxysmal atrial fibrillation. Circulation 2015; 132: 1311-9.

40. Chierchia GB, Capulzini L, Droogmans S, et al. Pericardial effusion in atrial fibrillation ablation: a comparison between cryoballoon and radiofrequency pulmonary vein isolation. Europace 2010; 12: 337-41. 